Original Research Paper

\title{
Recent Results on the Brittle Fracture of Terfenol-D Specimens under Magnetic Field
}

\author{
${ }^{1}$ Marco Colussi, ${ }^{2}$ Filippo Berto, ${ }^{3}$ Kotaro Mori and ${ }^{4}$ Fumio Narita \\ ${ }^{1}$ Department of Management and Engineering, University of Padua, Stradella S. Nicola 3, Vicenza 36100, Italy \\ ${ }^{2}$ Department of Engineering Design and Materials, NTNU, Richard Birkelands vei 2b, 7491 Trondheim, Norway \\ ${ }^{3}$ Department of Mechanical Engineering, Ibaraki University, Nakanarusawa-cho 4-12-1, Hitachi 316-8511, Japan \\ ${ }^{4}$ Department of Material Processing, Tohoku University, Aoba-yama 6-6-02, Sendai 980-8579, Japan
}

\author{
Article history \\ Received: 19-10-2016 \\ Revised: 19-11-2016 \\ Accepted: 27-12-2016 \\ Corresponding Author: \\ Filippo Berto \\ Department of Engineering \\ Design and Materials, NTNU, \\ Richard Birkelands vei $2 b$, \\ 7491 Trondheim, Norway \\ Email: filippo.berto@ntnu.no
}

\begin{abstract}
The following investigation has the purpose of describing, both experimentally and numerically, the fracture behavior of a giant magnetostrictive alloy commercially known as Terfenol-D. Single-edge precracked specimens have been analyzed via three-point bending tests, measuring fracture loads in the presence and absence of a magnetic field at various loading rates. The Strain Energy Density (SED), averaged in a finite control volume, has recently proved to be an excellent method of predicting brittle failures of cracked, $\mathrm{U}$ - and $\mathrm{V}$-notched specimens made out of different materials. The effects of the magnetic field and of the loading rate on Terfenol-D failures have been studied, as well as discussing the ability of SED criterion to seize these effects, by performing coupled-field finite element analyses. Finally, a relationship between the size of the SED's control volume and the loading rate has been proposed and failures have then been estimated in terms of averaged SED.
\end{abstract}

Keywords: Terfenol-D, Strain Energy Density, Magnetostriction, Fracture

\section{Introduction}

Magnetostriction is the variation of shape in a material when subjected to an external magnetic field. This effect was first delineated by the physicist James Joule in the 19th century. A considerable deformation in the presence of external magnetic fields has been displayed by the so-called giant magnetostrictive materials and forces applied on them can cause remarkable changes in the magnetization. One of the most widespread commercial materials for magnetostrictive applications is a rare earth element alloy of iron, terbium and dysprosium, named TerfenolD. For its renowned magnetostrictive elongation and its high energy density storing capacity at room temperature, granting it a large potential in many applications, this material has been raising much interest in the recent years (Engdahl, 1999). High power ultrasonic transducers make a large use of it, as well as automotive industry, avionics and robotics, which commonly use robust and responsive actuators and sensors based on the phenomenon of magnetostriction (Calkins et al., 2007). Terfenol-D has recently been used to produce sensors designed for stress monitoring of steel cables in suspended bridges (Zhang et al., 2014) and it might be employed in energy harvesting devices (Zhao and Lord, 2006; Li et al., 2010; Mori et al., 2015).

Terfenol-D is commonly known to be a brittle alloy, although its favorable applications, thus devices based on this material are sensitive to in-service fracture (Peterson et al., 1989). Hence, cracking and manufacturing induced defects could highly compromise the performances of the material. In addition, the literature provides little research on this topic, seemingly because of the high reactivity of the raw materials of Terfenol-D and its impurities, making it a hard to produce and very expensive alloy. These are the reasons behind the growing of interest in having a deeper mechanical knowledge about the sensitivity to defects of giant magnetostrictive materials, especially Terfenol-D.

In the last years professor Lazzarin and Zambardi (2001) has promoted a criterion based on Strain Energy Density (SED) to estimate brittle failures, taking place without any plastic deformation. The criterion asserts that brittle fracture failure occurs at the moment that the strain energy density, averaged in a circular control volume including a crack or notch tip, gets to a critical 
value dependent on the material. Afterwards this criterion has been deeply studied by many researchers, who proved it to be a reliable method in predicting brittle and high cycle fatigue failures of cracked, U- and Vnotched specimens composed of different materials, along with metals and ceramics (Berto et al., 2015; Berto and Lazzarin, 2014; 2009). Through the energy release rate (Narita et al., 2015) the effect of a magnetic field on fracture behavior of Terfenol-D has lately been studied, both experimentally and numerically, in order to attest that, in the absence of a magnetic field, the fracture resistance of the material is greater, under mode I loading condition, also showing its decrease when the magnetic field increases. This suggested that the increase of the energy release rate, induced by growing magnetic fields, might be the cause of the resistance decrease. In a recent investigation (Colussi et al., 2016) it has been shown that the SED criterion could be extended to the assessment of brittle behavior of giant magnetostrictive materials, under the same loading condition. Experimental data sets on fracture behavior of TerfenolD specimens under three-point bending have been extended in this study; in addition, fracture loads were measured in the presence and absence of the magnetic field and at different loading rates. The effects of the magnetic field and of the loading rate on Terfenol-D brittle failure have here been discussed, by performing coupled-field numerical analyses. The aim of this study has been the assessment of the capability of the SED criterion to seize these effects and, to this end, a relationship between the radius of the control volume and the loading rate has also been proposed.

\section{Analysis}

\section{Basic Equations}

The basic equations for magnetostrictive materials are here outlined. Considering a Cartesian coordinate system, $O-x_{1} x_{2} x_{3}$, the equilibrium equations are given by:

$$
\sigma_{j i, j}=0 ; \varepsilon_{i j k} H_{k, j}=0 ; B_{i, i}=0
$$

where, $\sigma_{i j}, H_{i}$ and $B_{i}$ are respectively the components of the stress tensor, the intensity vector of the magnetic field and the magnetic induction vector, whereas $\varepsilon_{i j k}$ is the Levi-Civita symbol. A comma followed by an index denotes partial differentiation with respect to the spatial coordinate $x_{i}$ and the Einstein's summation convention for repeated tensor indices is applied.

The constitutive laws are given as:

$$
\varepsilon_{i j}=s_{i j k l}^{H} \sigma_{k l}+d_{k i j} H_{k} ; B_{i}=d_{i k l} \sigma_{k l}+\mu_{i k}^{T} H_{k}
$$

where, $\varepsilon_{i j}$ are the components of the strain tensor and $s_{i j k l}^{H}, d_{i k l}, \mu_{i k}^{T}$ are respectively the magnetic field elastic compliance, the magnetoelastic constants and the magnetic permittivity.

Valid symmetry conditions are:

$$
s_{i j k l}^{H}=s_{j i k l}^{H}=s_{i j l k}^{H}=s_{k l i j}^{H} ; d_{k i j}=d_{k j i} ; \mu_{i j}^{T}=\mu_{j i}^{T}
$$

The relation between the strain tensor and the displacement vector $u_{i}$ is:

$\varepsilon_{i j}=\frac{1}{2}\left(u_{j, i}+u_{i, j}\right)$

The magnetic field intensity, named $\varphi$ the potential, is written as:

$H_{i}=\varphi_{, i}$

For Terfenol-D, the constitutive relations can be written as:

$$
\begin{aligned}
& \left\{\begin{array}{c}
\varepsilon_{11} \\
\varepsilon_{22} \\
\varepsilon_{33} \\
2 \varepsilon_{23} \\
2 \varepsilon_{31} \\
2 \varepsilon_{12}
\end{array}\right\}=\left[\begin{array}{cccccc}
s_{11}^{H} & s_{12}^{H} & s_{13}^{H} & 0 & 0 & 0 \\
s_{12}^{H} & s_{11}^{H} & s_{13}^{H} & 0 & 0 & 0 \\
s_{13}^{H} & s_{13}^{H} & s_{33}^{H} & 0 & 0 & 0 \\
0 & 0 & 0 & s_{44}^{H} & 0 & 0 \\
0 & 0 & 0 & 0 & s_{44}^{H} & 0 \\
0 & 0 & 0 & 0 & 0 & s_{66}^{H}
\end{array}\right]\left\{\begin{array}{c}
\sigma_{11} \\
\sigma_{22} \\
\sigma_{33} \\
\sigma_{23} \\
\sigma_{31} \\
\sigma_{12}
\end{array}\right\} \\
& +\left[\begin{array}{ccc}
0 & 0 & d_{31} \\
0 & 0 & d_{31} \\
0 & 0 & d_{33} \\
0 & d_{15} & 0 \\
d_{15} & 0 & 0 \\
0 & 0 & 0
\end{array}\right]\left\{\begin{array}{l}
H_{1} \\
H_{2} \\
H_{3}
\end{array}\right\}
\end{aligned}
$$

$\left\{\begin{array}{l}B_{1} \\ B_{2} \\ B_{3}\end{array}\right\}=\left[\begin{array}{cccccc}0 & 0 & 0 & 0 & d_{15} & 0 \\ 0 & 0 & 0 & d_{15} & 0 & 0 \\ d_{31} & d_{31} & d_{33} & 0 & 0 & 0\end{array}\right]\left\{\begin{array}{l}\sigma_{11} \\ \sigma_{22} \\ \sigma_{33} \\ \sigma_{23} \\ \sigma_{31} \\ \sigma_{12}\end{array}\right\}$

$$
+\left[\begin{array}{ccc}
\mu_{11}^{T} & 0 & 0 \\
0 & \mu_{11}^{T} & 0 \\
0 & 0 & \mu_{33}^{T}
\end{array}\right]\left\{\begin{array}{l}
H_{1} \\
H_{2} \\
H_{3}
\end{array}\right\}
$$

where:

$$
\left.\begin{array}{c}
\sigma_{23}=\sigma_{32} ; \sigma_{31}=\sigma_{13} ; \sigma_{12}=\sigma_{21} \\
\varepsilon_{23}=\varepsilon_{32} ; \varepsilon_{31}=\varepsilon_{13} ; \varepsilon_{12}=\varepsilon_{21}
\end{array}\right\}
$$




$$
\begin{gathered}
\left.\begin{array}{c}
s_{11}^{H}=s_{1111}^{H}=s_{2222}^{H} ; s_{12}^{H}=s_{1122}^{H} \\
s_{13}^{H}=s_{1133}^{H}=s_{2233}^{H} ; s_{33}^{H}=s_{3333}^{H} \\
s_{44}^{H}=4 s_{2323}^{H}=4 s_{3131}^{H} \\
s_{66}^{H}=4 s_{1212}^{H}=2\left(s_{11}^{H}-s_{12}^{H}\right)
\end{array}\right\} \\
\left.\begin{array}{c}
d_{15}=2 d_{131}=2 d_{223} \\
d_{31}=d_{311}=d_{332} \\
d_{33}=d_{333}
\end{array}\right\}
\end{gathered}
$$

The relationship between magnetostriction and magnetic field intensity is non-linear. Nonlinearity arises from the movement of the magnetic domain walls (Wan et al., 2003). To take into account this behavior, the constants $d_{15}, d_{31}$ and $d_{33}$ are written as function of second order magnetoelastic constants:

$$
\left.\begin{array}{c}
d_{15}=d_{15}^{m} \\
d_{31}=d_{31}^{m}+m_{31} H_{z} \\
d_{33}=d_{33}^{m}+m_{33} H_{z}
\end{array}\right\}
$$

where, $d_{15}^{m}, d_{31}^{m}$ e $d_{33}^{m}$ are the piezomagnetic constants, whereas $m_{31}$ and $m_{33}$ are the second order magnetoelastic constants.

\section{Averaged Strain Energy Density (SED)}

According to Lazzarin and Zambardi (2001), the brittle failure of a component occurs when the total strain energy, $\bar{W}$, averaged in a specific control volume located at a notch or crack tip, reaches the critical value $W_{c}$. In agreement with Beltrami (1885), named $\sigma_{t}$ the ultimate tensile strength under elastic stress field conditions and $E$ the Young's modulus of the material, the critical value of the total strain energy can be determined by the relation:

$$
W_{c}=\frac{\sigma_{t}^{2}}{2 E}
$$

The control volume takes different shapes based on the considered kind of notch. If the notch is represented by a crack, its opening angle is equal to zero and the control volume is circular and centered on the crack tip. Being this the case, the radius $R_{c}$ of the control volume can be evaluated by means of the following expression (Yosibash et al., 2004):

$$
R_{c}=\frac{(1+v)(5-8 v)}{4 \pi}\left(\frac{K_{I C}}{\sigma_{t}}\right)^{2}
$$

where, $K_{l c}$ is the material fracture toughness, $v$ is the Poisson's ratio and $\sigma_{t}$ is the ultimate tensile strength of an un-notched specimen. If the material fracture toughness is not known, an empirical approach can then be a good alternative for determining $R_{c}$, as described in the following sections.

\section{Finite Element Model}

The strain energy density averaged in the control volume, $\bar{W}$, can be computed directly by means of a finite element analysis. Analyses were performed by means of ANSYS R14.5 finite element software, both in plane strain and plane stress conditions depending on the specimens' width. For this purpose, solid models were used to determine the most appropriate condition. The basic equations for magnetostrictive materials are mathematically equivalent to those of the piezoelectric materials, as shown by Tiersten (1969), so four nodes PLANE13 and eight nodes SOLID5 coupled-field solid elements from ANSYS library were used, respectively for plane and solid models and the magnetic field has been introduced by a voltage difference. Figure 1 shows the schematic representation of the boundary conditions and the adopted reference system. The coordinate axes $x$ $=x_{1}$ and $z=x_{3}$ are chosen such that the $y=x_{2}$ axis coincides with the thickness direction and such that the easy axis of magnetization is the $z$-direction. Because of symmetry, only half of the model was used.

The mesh adopted to compute $\bar{W}$ had the same grade of refinement adopted in a previous work by the present authors (Colussi et al., 2016), in which models with 6400 elements were used to evaluate the energy release rate by means of J-integral on the same geometry.

\section{Experimental Tests}

The alloy known by the commercial name of Terfenol-D $\left(\mathrm{Tb}_{0.3} \mathrm{Dy}_{0.7} \mathrm{Fe}_{1.9}\right)$, supplied by Etrema Products, Inc. (USA), has been chosen among giant magnetostrictive materials to perform all tests. The material properties are listed in Table 1.

The testing apparatus is illustrated in Fig. 2: Singleedge precracked specimens have been subjected to threepoint bending, in the presence and absence of the magnetic field and at different loading rates, in order to measure the fracture load, $P_{c}$.

The following Fig. 3 depicts one of the tested specimens, which were $5 \mathrm{~mm}$ thick, $3 \mathrm{~mm}$ wide and 15 $\mathrm{mm}$ long. By utilizing a tungsten cutter, a $0.5 \mathrm{~mm}$ deep crack was produced on one side of all the specimens before testing, thus introducing a weak point.

The specimens, simply supported with span of 13 $\mathrm{mm}$, have been loaded at their midpoint via a $250 \mathrm{~N}$ load cell (resolution: $0.01 \mathrm{~N}$ ), for various loading rates: $0.05,0.5$ and $3.0 \mathrm{Ns}^{-1}$. An electromagnet has applied in the longitudinal direction a uniform magnetic field, with magnetic induction $B_{0}$. A magnetic induction of $0.03 \mathrm{~T}$ has been applied in all 
tests and chosen as a representative value, considering that the common range of magnetic induction which Terfenol-D devices usually work in varies from 0.02 $\mathrm{T}$ to $0.05 \mathrm{~T}$. It is worth noting that, since some of the constituent elements of Terfenol-D are two very expensive rare earths like terbium and dysprosium, only two to three specimens at each condition were prepared for testing.

The non-linear trend of Terfenol-D magnetostriction versus magnetic field has been assessed using an experimental procedure. Let us consider a Cartesian coordinate system, $O-x y z$, whose origin is located at the top center of an uncracked specimen. The longitudinal magnetostriction (in the $z$-direction) appears to be predominant, as the specimen's dimension in this direction is over three times greater than the other two dimensions: That is why it is reasonable to assume $m_{31}$ equal to zero (Jia et al., 2006). By means of a strain gauge located at $x=y=z=0 \mathrm{~mm}$, the trend of magnetostriction has been measured modulating the magnetic field intensity in the $z$-direction. A comparison between the measured strain $\varepsilon_{z z}$ and the one obtained numerically has been done, revealing a proper value for the second order magnetoelastic constant $m_{33}$ of $4.82 \times 10^{-12} \mathrm{~m}^{2} \mathrm{~A}^{-2}$, used in the analyses to compute the $S E D$. The trend of strain versus applied magnetic field is shown below (Fig. 4): The full dots stand for the experimental data, while the solid line represents the numerical trend, having considered the second order magnetoelastic constant.

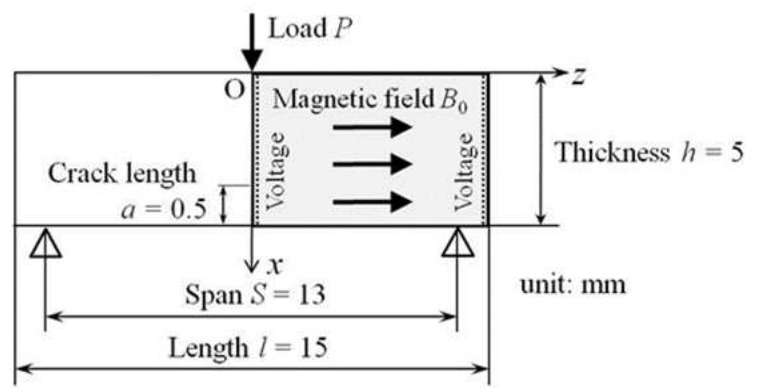

Fig. 1. Schematic representation of the model and boundary conditions

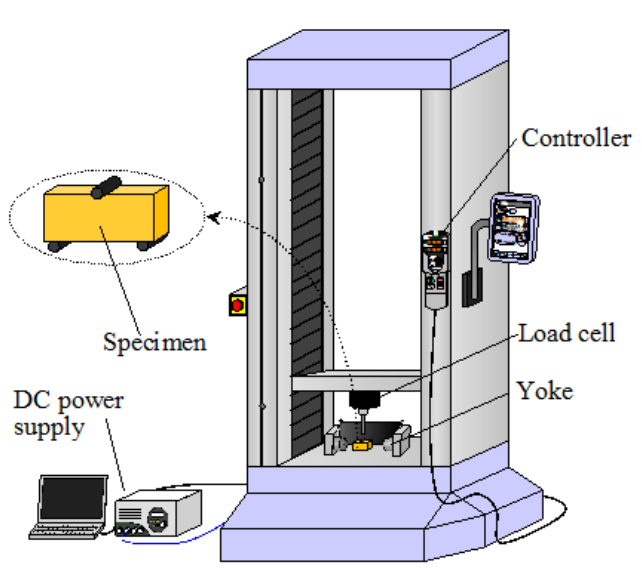

Fig. 2. Schematic representation of the experimental setup

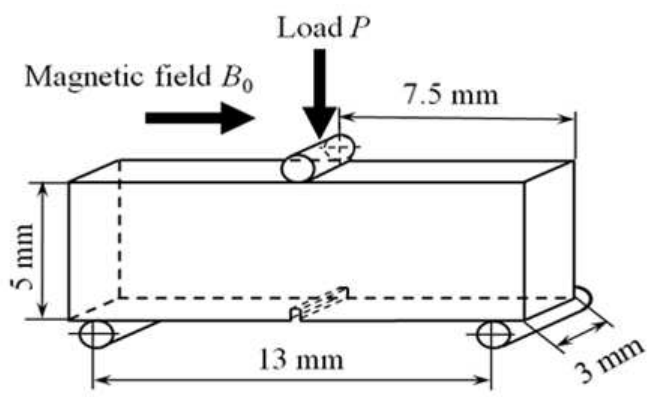

Fig. 3. Specimen's geometry and testing condition

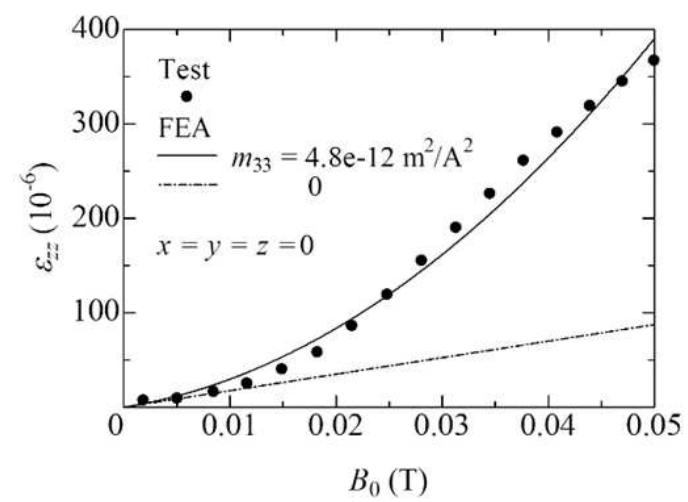

Fig. 4. Trend of strain versus magnetic field in Terfenol-D

Table 1. Terfenol-D material properties

\begin{tabular}{|c|c|c|c|c|c|}
\hline \multicolumn{5}{|c|}{ Elastic compliance $\left[10^{-12} \mathrm{~m}^{2} \mathrm{~N}^{-1}\right]$} & \multirow{2}{*}{$\begin{array}{l}\text { Density } \\
{\left[\mathrm{kg} \mathrm{m}^{-3}\right]} \\
\rho\end{array}$} \\
\hline$s_{11}^{H}$ & $s_{22}^{H}$ & $s_{44}^{H}$ & $s_{12}^{H}$ & $s_{13}^{H}$ & \\
\hline 17.9 & 17.9 & 26.3 & -5.88 & -5.88 & 9250 \\
\hline \multicolumn{3}{|c|}{ Piezo-magnetic constants $\left[10^{-9} \mathrm{~mA}^{-1}\right]$} & \multicolumn{3}{|c|}{ Magnetic permeability $\left[10^{-6} \mathrm{Hm}^{-1}\right]$} \\
\hline$d_{31}^{m}$ & $d_{33}^{m}$ & $d_{15}^{m}$ & $\mu_{11}^{T}$ & $\mu_{33}^{T}$ & \\
\hline-5.3 & 11 & 28 & 6.29 & 6.29 & \\
\hline
\end{tabular}




\section{Results}

The following table contains the fracture loads, $P_{c}$, experimentally measured at each loading rate, in the presence and absence of the magnetic field: Bold numbers represent the average value at each condition, whereas numbers in brackets represent the relative standard deviations.

Figure 5 shows below the average fracture loads. Maximum and minimum values of $P_{c}$ are designated with the error bars. It has been noted that, in the presence of the magnetic field, the average fracture loads at 0.05 , 0.50 and $3.0 \mathrm{Ns}^{-1}$ are decreased respectively about $7 \%$, $9 \%$ and $14 \%$.

Furthermore, Terfenol-D has shown a decrease in fracture load with a decrease in the loading rate, as other materials such as TiAl alloys (Cao et al., 2007) and piezoelectric ceramics (Shindo et al., 2009; Narita et al., 2012) have exhibited a similar behavior.

It is here assumed that the critical radius $R_{c}$, depending on the material, varies with the same speed which the load is applied at, given that Terfenol-D material properties are determined by the loading rate. The mean values of critical loads in Table 2 , in the presence and absence of the magnetic field, have been used to plot the related averaged $S E D$ as a function of the control volume radius, allowing to identify various intersections for each loading rate. These intersections have been found at $0.050,0.056$ and $0.1 \mathrm{~mm}$ respectively for the loading rates $0.05,0.5$ and 3.0 $\mathrm{Ns}^{-1}$. Evidence shows that, at the critical load, the value of the strain energy density of the material, averaged in a control volume with size varying accordingly to the loading rate, appears to be independent of the ratio between the applied load and the magnetic field. The relation between $R_{c}$ and the loading rate has shown to be adequately interpreted by a linear model and, after that, the following relationship (evaluated for loading rates from 0.05 to $3.0 \mathrm{Ns}^{-1}$ ) has been proposed, using a simple linear regression model:

$$
R_{c}=0.0195 \cdot \frac{d P}{d t}+0.05
$$

It has been found that the critical radius of 0.07 $\mathrm{mm}$, obtained from Equation 13 and recommended in a previous work (Colussi et al., 2016) without considering the loading rate, falls within the range of variation here proposed. In the next figure (Fig. 6) experimental data in terms of the square root of the ratio between the averaged strain energy density, $\bar{W}$ and the critical value of strain energy, $W_{c}$, are displayed. It is worth pointing out that this parameter has been chosen because of its proportionality to the fracture load. A critical strain energy equal to 0.02 $\mathrm{MJm}^{-3}$ is assumed, obtained from Equation 12, assuming Young's modulus equal to $30 \mathrm{GPa}$, Poisson's ratio equal to 0.25 and tensile strength equal to 34 $\mathrm{MPa}$, since these are the medium characteristics provided by the material supplier (Etrema Products, Inc.). Moreover, in control volumes having radius given by Equation 14, the averaged strain energy density, $\bar{W}$, has been calculated. The assumption made on the Young's modulus to consider it as independent of the applied magnetic field is allowed in the range of variation of $B_{0}$. A summary of experimental data from a previous work (Narita et al., 2015) is presented in Fig. 6, displaying fracture loads measured under three-point bending at the loading rates of $0.2 \mathrm{Ns}^{-1}$ and $3.0 \mathrm{Ns}^{-1}$, in the presence and absence of the $0.03 \mathrm{~T}$ magnetic field.

Table 2. Measured fracture loads as a function of the loading rate and the magnetic field

\begin{tabular}{lll}
\hline & $\mathrm{Pc}[\mathrm{N}]$ & \\
$\mathrm{dP} / \mathrm{dt}$ & $---0 \mathrm{~T}$ & $\mathrm{~B}_{0}=0.03 \mathrm{~T}$ \\
\hline $0.05 \mathrm{Ns}^{-1}$ & 58.3 & 59.2 \\
& 65.8 & 61.9 \\
& 74.7 & 64.6 \\
$0.5 \mathrm{Ns}^{-1}$ & $66.3(5.81)$ & $61.9(1.91)$ \\
& 66.6 & 60.7 \\
$3.0 \mathrm{Ns}^{-1}$ & 68.5 & 61.6 \\
& $67.5(0.78)$ & $61.1(0.37)$ \\
& 71.0 & 74.2 \\
& 79.2 & 59.3 \\
& - & 60.0 \\
\hline
\end{tabular}

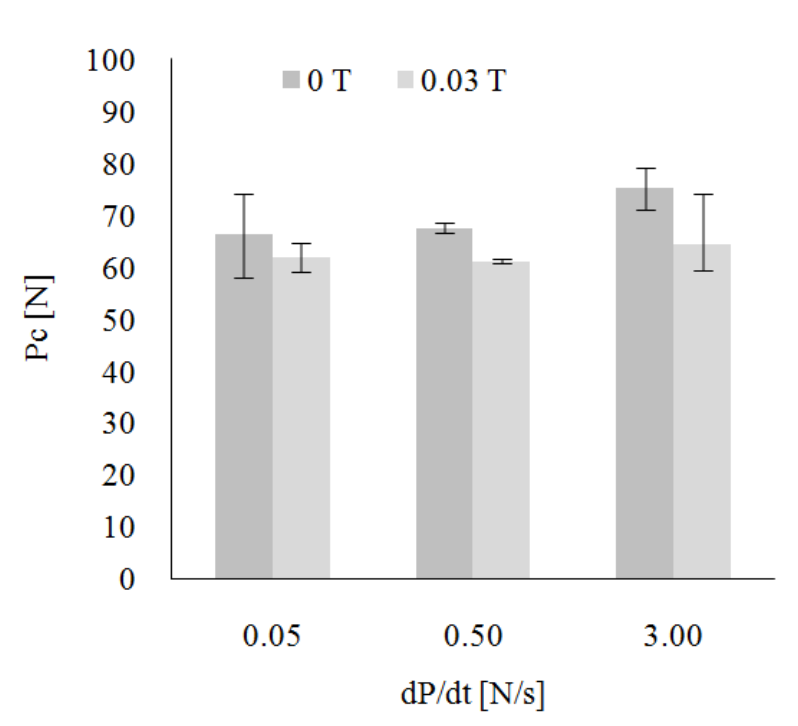

Fig. 5. Mean fracture loads as a function of the loading rate and the magnetic field $\mathrm{B}_{0}$ 


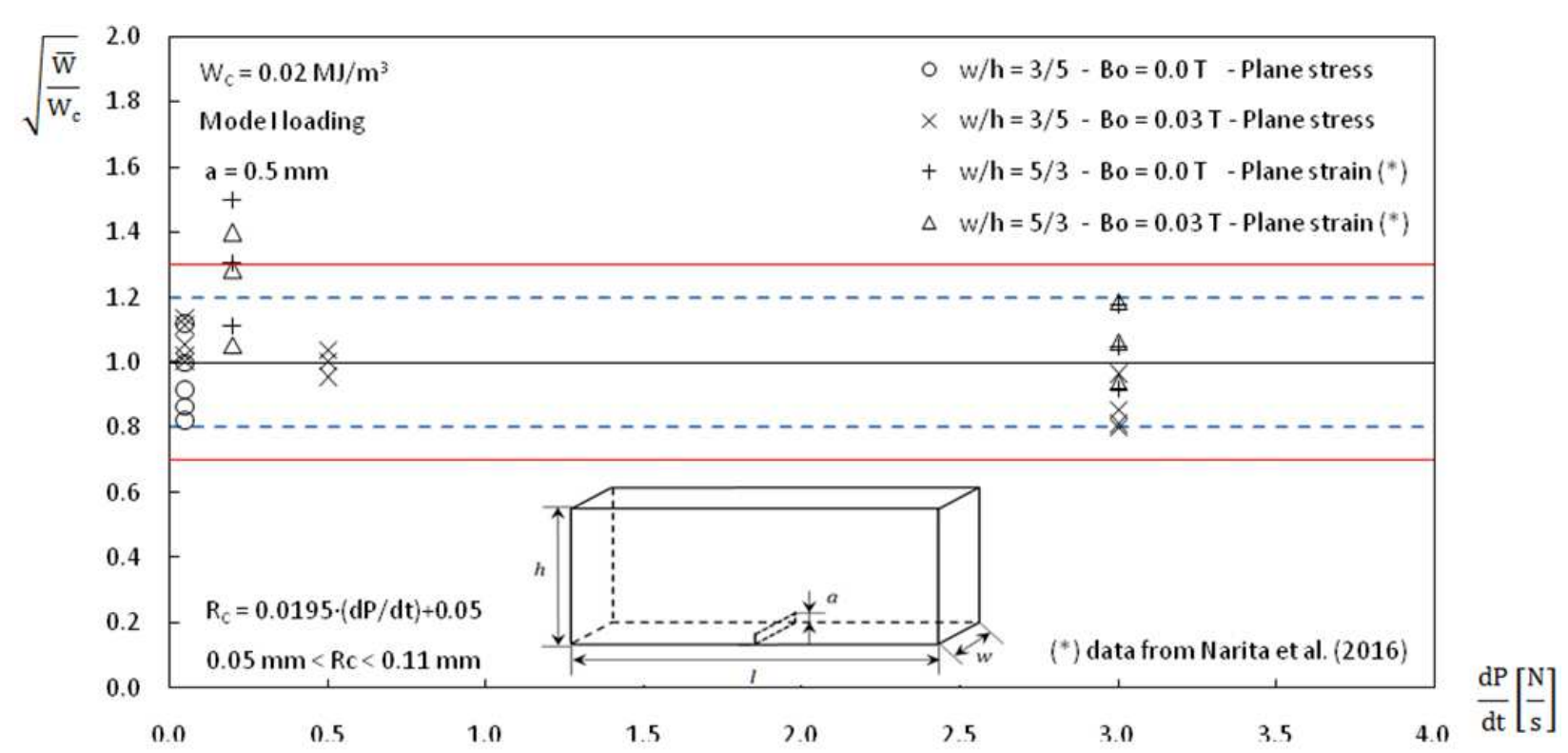

Fig. 6. Summary from specimens made out of Terfenol-D at various loading rates in the presence and absence of the magnetic field $\mathrm{B}_{0}$

Specimens were $3 \mathrm{~mm}$ thick, $5 \mathrm{~mm}$ wide and $15 \mathrm{~mm}$ long. The crack depth was $0.5 \mathrm{~mm}$. For their finite element modeling, considering the different geometry (ratio between width, $w$ and thickness, $h$, equal to $5 / 3$ instead of $3 / 5$ ), the plane strain condition was preferred as more suitable over the plane stress one. Evidence showed that almost all experimental data are contained in a confined scatter band, limited from 0.80 to 1.20 (4 data over 35 being outside of this range). However, the few data exceeding the band fall in the safety region of the plot. In conclusion, being the scatter of the data quite limited, the averaged $S E D$ appears to be an appropriate criterion for the fracture strength assessment of Terfenol-D alloy cracked specimens, under mode I condition, in the presence or absence of the magnetic field and with variable loading rate. In the authors' opinion this encouraging result leads to believe in the SED suitability to reliably assess Terfenol-D brittle failure. The relationship here proposed between the size of the control volume and the loading rate also allows to take into account the loading rate through finite element analyses.

\section{Conclusion}

The sensitivity of the rare earth element Terfenol-D alloy to defects was investigated both numerically and experimentally. Terfenol-D fracture resistance has shown to be greater in the absence of the magnetic field under three-point bending condition. The increase of the strain energy density around the crack tip, when the magnetic field is acting, validates this behavior. It has also been verified the failure of Terfenol-D at decreasing fracture loads as the loading rate decreases. Assuming a linear relationship between the size of the control volume and the loading rate, the averaged SED criterion is able to seize this behavior and seems capable of predicting Terfenol-D failures.

\section{Acknowledgement}

The authors gratefully acknowledge Alberto Zordan for his helpful contribution in revising the manuscript.

\section{Author's Contributions}

Marco Colussi: Performed the finite element analyses and wrote the most of the manuscript.

Filippo Berto: Coordinated the research project and contributed to the writing of the manuscript.

Kotaro Mori and Fumio Narita: Performed the experimental tests and contributed to the writing of the manuscript.

\section{Ethics}

The authors declare no conflict of interest and no ethical issues that may arise after the publication of this manuscript.

\section{References}

Beltrami, E., 1885. Sulle condizioni di resistenza dei corpi elastici. Rendiconti Del Regio Istituto Lombardo. 18: 704-714. DOI: 10.1007/BF02824697 
Berto, F., A. Campagnolo and P. Gallo, 2015. Brittle failure of graphite weakened by v-notches: A review of some recent results under different loading modes. Strength Mater., 47: 488-506.

DOI: $10.1007 / \mathrm{s} 11223-015-9682-7$

Berto, F. and P. Lazzarin, 2009. A review of the volumebased strain energy density approach applied to Vnotches and welded structures. Theoret. Applied Fract. Mechan., 52: 183-194.

DOI: $10.1016 /$ j.tafmec.2009.10.001

Berto, F. and P. Lazzarin, 2014. Recent developments in brittle and quasi-brittle failure assessment of engineering materials by means of local approaches. Mater. Sci. Eng. R, 75: 1-48.

DOI: $10.1016 /$ j.mser.2013.11.001

Calkins, F., A.B. Flatau and M.J. Dapino, 2007. Overview of magnetostrictive sensor technology. J. Intell. Mater. Syst. Struct., 18: 1057-1066. DOI: $10.1177 / 1045389 X 06072358$

Cao, R., M.X. Lei, J.H. Chen and J. Zhang, 2007. Effects of loading rate on damage and fracture behavior of TiAl alloys. Mater. Sci. Eng. A, 465: 183-193. DOI: 10.1016/j.msea.2007.02.026

Colussi, M., F. Berto, K. Mori and F. Narita, 2016. Fracture behavior of cracked giant magnetostrictive materials in three-point bending under magnetic fields: Strain energy density criterion. Adv. Eng. Mater. DOI: 10.1002/adem.201500533

Engdahl, G., 1999. Handbook of Giant Magnetostrictive Materials. 1st Edn., Academic Press, New York, ISBN-10: 0080533604, pp: 386.

Jia, Z., W. Liu, Y. Zhang, F. Wang and D. Guo, 2006. A nonlinear magnetomechanical coupling model of giant magnetostrictive thin films at low magnetic fields. Sensors Actuat. A, 128: 158-164.

DOI: $10.1016 /$ j.sna.2006.01.018

Lazzarin, P. and R. Zambardi, 2001. A finite-volumeenergy based approach to predict the static and fatigue behavior of components with sharp Vshaped notches. Int. J. Fract., 112: 275-298. DOI: 10.1023/A:1013595930617

Li, P., Y. Wen, P. Liu, X. Li and C. Jia, 2010. A magnetoelectric energy harvester and management circuit for wireless sensor network. Sensors Actuat. A, 157: 100-106. DOI: 10.1016/j.sna.2009.11.007

Mori, K., T. Horibe, S. Ishikawa, Y. Shindo and F. Narita, 2015. Characteristics of vibration energy harvesting using giant magnetostrictive cantilevers with resonant tuning. Smart Mater. Struct., 24: $125032-125032$.

DOI: $10.1088 / 0964-1726 / 24 / 12 / 125032$
Narita, F., Y. Morikawa, Y. Shindo and M. Sato, 2012. Dynamic fatigue behavior of cracked piezoelectric ceramics in three-point bending under AC electric fields. J. Eur. Ceramic Society, 32: 3759-3766.

DOI: 10.1016/j.jeurceramsoc.2012.05.033

Narita, F., K. Shikanai, Y. Shindo and K. Mori, 2015. Three-point bending fracture behavior of cracked giant magnetostrictive materials under magnetic fields. J. Test. Evaluat., 44: 1454-1460.

DOI: $10.1520 /$ JTE20140439

Peterson, D.T., J.D. Verhoeven, O.D. McMasters and W.A. Spitzig, 1989. Strength of terfenol-D. J. Applied Phys., 65: 3712-3713.

Shindo, Y., F. Narita, K. Mori and T. Nakamura, 2009. Nonlinear bending response of giant magnetostrictive laminated actuators in magnetic fields. J. Mechan. Mater. Struct., 4: 941-949.

DOI: 10.2140/jomms.2009.4.941

Tiersten, H.F., 1969. Linear Piezoelectric Plate Vibrations: Elements of the Linear Theory of Piezoelectricity and the Vibrations of Piezoelectric Plates. 1st Edn., Springer, New York, ISBN-10: 1489962212, pp: 212.

Wan, Y., D. Fang and K.C. Hwang, 2003. Non-linear constitutive relations for magnetostrictive materials. Int. J. Non-Linear Mechan., 38: 1053-1065. DOI: 10.1016/S0020-7462(02)00052-5

Yosibash, Z., A.R. Bussiba and I. Gilad, 2004. Failure criteria for brittle elastic materials. Int. J. Fract., 125: 307-333.

DOI: 10.1023/B:FRAC.0000022244.31825.3b

Zhang, R., Y. Duan, S.W. Or and Y. Zhao, 2014. Smart Elasto-Magneto-Electric (EME) sensors for stress monitoring of steel cables: Design theory and experimental validation. Sensors, 14: 13644-13660. DOI: $10.3390 / \mathrm{s} 140813644$

Zhao, X. and D.G. Lord, 2006. Application of the Villari effect to electric power harvesting. J. Applied Phys., 99 08M703-08M703-3.

DOI: $10.1063 / 1.2165133$

\section{Nomenclature}

a Crack depth for cracked specimens

B Magnetic induction vector

$B_{i} \quad i$ component of the magnetic induction

$d_{k i j} \quad$ Magnetoelastic constants

E Young's modulus

$G \quad$ Strain energy release rate

$G_{c} \quad$ Critical strain energy release rate

$h \quad$ Thickness of the specimens 
$\boldsymbol{H} \quad$ Intensity vector of the magnetic field

$H_{i} \quad i$ component of the intensity vector of the magnetic field

$J \quad$ J-integral value

$l \quad$ Length of the specimens

$K_{\mathrm{I}} \quad$ Mode I stress intensity factor

$K_{\text {IC }} \quad$ Material fracture toughness

n Exit path normal

$R_{\mathrm{c}} \quad$ Radius of the control volume

$s_{i j k l}^{H} \quad$ Elastic compliance

$\boldsymbol{T}$ Surface tension vector

$\boldsymbol{u}$ Displacement vector

$u_{i} \quad i$ component of the displacement vector

$w \quad$ Width of the specimens
$W \quad$ Strain energy density

$\bar{W} \quad$ Averaged strain energy density

$W_{c} \quad$ Critical strain energy

$W_{m} \quad$ Magnetic enthalpy

Greek

$\varepsilon \quad$ Strain tensor

$\varepsilon_{i j} \quad i j$ component of the strain tensor

$\mu_{i j}^{T} \quad$ Magnetic permittivity

$v$ Poisson's ratio

$\sigma \quad$ Cauchy stress tensor

$\sigma_{i j} \quad i j$ component of the stress tensor

$\sigma_{t} \quad$ Tensile strength

$\varphi \quad$ Magnetic potential

$\Omega \quad$ Area of the control volume 